\title{
Chlorhexidine in Dentistry - Five Decades and Continuing - Where are we Now?
}

\section{Dr. Krishna Madhukumaran Pillai Sreekaladevi MDS ${ }^{1}$, Valliammai Rajendran MDS $^{2 *}$, Dr. Veenadevi Thonthula BDS ${ }^{3}$, Jennifer Joseph Antony MDS $^{4}$ and Dr. Shreya Kothari BDS MPH ${ }^{5}$}

${ }^{1}$ Ex-Post Graduate, Department of oral medicine and maxillofacial radiology, KVG dental college and hospital, Sulliya

${ }^{2}$ Ex-post Graduate, Department of Periodontology, Manipal College of Dental

Sciences, Mangalore, Manipal Academy of Higher Education (MAHE), Manipal,

Karnataka, India

${ }^{3} 313$ orchards Boulevard SW, Edmonton AB T6X1Y9, Canada

${ }^{4}$ Periodontist, 5/14 Mcilwraith Street, Everton Park QLD 4053, Australia

${ }^{5}$ Atlanta, Georgia, USA

*Corresponding Author: Valliammai Rajendran, Ex-post Graduate, Department of Periodontology, Manipal College of Dental Sciences, Mangalore, Manipal Academy of Higher Education (MAHE), Manipal, Karnataka, India.
Received: October 05, 2021

Published: October 26, 2021

(C) All rights are reserved by Valliammai

Rajendran., et al.

\begin{abstract}
Chlorhexidine(CHX) has a wide variety of uses in the management of oral diseases. It is a cationic bisbiguanide with broad antibacterial activity, and a wide spectrum of activity encompassing gram-positive and gram-negative bacteria, yeasts, dermatophytes, and viruses (enveloped). CHX is a commonly used antiseptic mouthwash due to its antimicrobial effects. It is commonly preferred among dentists. The clinical effect of CHX is likely due to both its substantive and antibacterial properties. Search terms were entered into MEDLINE, PubMed, Google Scholar, and the Cochrane database to find evidence about CHX use in the oral cavity. It plays a key role in dentistry and is used to treat or prevent periodontal disease, and has earned its eponym of the gold standard. The aim of this article was to provide a detailed review of the current uses of CHX in dentistry in terms of the management of oral diseases, highlighting the need for further studies to support its safe and appropriate use.
\end{abstract}

Keywords: Chlorhexidine; Mouthwash; Oral Hygiene

\section{Introduction}

Chlorhexidine(CHX) gluconate is a bisbiguanide compound consisting of four chlorophenyl rings and two biguanide groups connected by a central hexamethylene bridge [1]. It is strongly basic and $\mathrm{pH}$ is $>3.5$. It is a gluconate salt that has been in use since the mid-19th century. It was developed in the 1940s by Imperial chemical Industries, England. It has been used as an adjunct for a variety of oral diseases. It was first used for plaque control by Schroeder in 1969 [2]. It's a broad-spectrum anti-microbial agent. It is also used for the disinfection of non-living clinical surfaces and catheters. It is one of the most widely used chemicals in the field of Dentistry. CHX is available in 3 forms: digluconate, acetate, and hydrochloride. The latter is less water-soluble than the other forms. The available formulations of CHX are gels, chips, sprays, varnishes, 
and mouthwashes [3]. Its high substantivity makes it stand out compared to other chemical compounds [4]. Hence the focus of this article is to provide insight views on chlorhexidine as a single solution for various oral diseases. Search terms were entered into MEDLINE, PubMed, Google Scholar, and the Cochrane database. Evidences collected are given importance based on the hierarchy.

\section{Anti-microbial effect}

CHX is an effective membrane-type anti-microbial agent with an effect on the inner cytoplasmic membrane [5]. It acts against grampositive and negative bacteria, yeast, viruses, fungi, and dermatophytes [6]. It anti-bacterial effects are due to its ability to alter the permeability of the cell membrane. It could be either bactericidal or bacteriostatic based on its concentration. At low concentrations $(0.02 \%), \mathrm{CHX}$ has its effects in displacing $\mathrm{Ca} 2+$ and $\mathrm{Mg} 2+$ ions along with loss of $\mathrm{K}+$ ions from the cell wall which is the bacteriostatic property of the chemical compound. At higher concentrations such as $0.1 \%$, it causes the intracellular components of the cell to leak out eventually followed by cell death which shows the effective bactericidal activity of the compound $[7,8]$. CHX is great against oral biofilm. It inhibits pellicle formation and prevents protein adsorption to the tooth surface. Then CHX binds to the bacteria, displaces $\mathrm{Ca} 2+$ ions, preventing biofilm formation. CHX has similar effects on enveloped viruses. Its efficacy is less on non-enveloped viruses such as the human papillomavirus [9]. Its anti-fungal effect accounts to a decrease in the number of fungi residing in oral mucosa, teeth, and dentures rather than the fungicidal effect [10].

\section{Uses in oral diseases}

CHX has a wide variety of usefulness in oral health care. It is used in the management of oral hygiene, dental plaque, dental caries, gingivitis, periodontitis, peri-implantitis, irrigating agent, pre-procedural mouth rinse, and management of oral mucosal diseases. It is available as over-the-counter (OTC) and available to the common public at an affordable cost. The upcoming sections explain the use of CHX in oral diseases.

\section{Dental caries}

Dental caries is caused by the biofilm buildup on the tooth surface leading to the production of lactic acid and dissolution of tooth structure. Hence it is thought that the use of CHX mouth rinse $(0.2 \%)$ could potentially reduce plaque formation and even- tually results in a low incidence of caries [11]. Although CHX inhibits plaque formation, Cochrane review states that the use of CHX $(0.2 \%)$ did not significantly result in a reduced number of caries incidence [12]. In addition, CHX varnish has been tried to see if it results in a reduction of caries. However, it did not result in caries reduction [13]. $0.05 \%$ sodium fluoride mouth rinses are suggested for a reduction in dental caries [14].

\section{Gingival and periodontal diseases}

The presence of bacteria in dental plaque or oral biofilm produces host inflammatory response of gingiva and periodontium resulting in gingivitis and periodontitis respectively. CHX is used as a mouthwash against plaque-producing bacteria [15]. It should always be used along with mechanical plaque control measures such as tooth brushing and interdental aids, as an adjunct, as suggested by the European Federation of Periodontology (EFP) [16]. Reduction in clinical signs of gingivitis and periodontitis has been shown in studies with the use of $0.2 \%$ CHX mouthwash for 4-6 weeks [17]. When CHX is used as a mouth wash, it should be used 30 minutes to 2 hours after tooth brushing as it could interact with the anionic compounds of the toothpaste and it could nullify the effect of fluoride that is present in toothpaste [18].

Apart from use as a mouthwash, CHX is employed as a local drug delivery agent in shallow periodontal pockets as an adjunct to mechanical debridement. Periochip has been demonstrated in the reduction of probing pocket depth (PPD) and clinical attachment loss (CAL) in periodontitis cases. Though the amount of reduction in PPD and CAL is less, CHX has effects in the management of periodontal diseases [19]. Cochrane reviews suggest that CHX has not been effective even as an adjunct in cases of moderate and severe periodontitis as disease-causing anaerobic bacteria lies in deep periodontal pockets in such cases. However, it is still effective in mild cases of periodontitis [20,21].

CHX is also suggested for the management of necrotizing periodontal diseases. Poor oral hygiene is the major contributing factor in the course of these diseases. Gingiva becomes swollen with associated sloughing and halitosis. Oral hygiene maintenance stays as the first line of treatment. Apart from this, systemic administration of antibiotics and the use of $\mathrm{CHX}(0.12 \%$ or $0.2 \%)$ or hydrogen peroxide mouthwash (6\%) is used as an adjunctive aid [15]. This could 
be because of the anti-bacterial nature of CHX against P. intermedia which is considered as a prime causative agent in necrotizing periodontal diseases [22].

\section{Peri-implantitis}

The wide range of use of CHX extends to the management of peri-implant diseases as well. CHX mouthwash has effects in reduction of microbial load if used 7-10 days prior to placement of implant which results in a significant reduction of postoperative complications. Postoperatively, CHX is used as a local agent on the surgical site for betterment in healing and also as a mouthwash [23]. This helps in the reduction of dental biofilm on the surface of the implant. In cases of peri-implantitis, CHX is used as a gel and irrigating agent for microbial load reduction [23]. Administration of periochip resulted in a reduction of pocket depth around implants. However, systematic review suggests that clinical signs such as bleeding on probing and pocket depth are not improved with the adjunctive use of CHX when compared with mechanical debridement alone [24]. Other studies suggest a reduction of microbial load with the use of CHX in peri-implantitis cases whereas clinical signs did not improve significantly $[23,25]$.

\section{Root canal irrigant}

Proper disinfection of the root canal is a significant factor in determining the success of root canal treatment. Various root canal irrigants are available in use. It is found that CHX when used as a root canal irrigant, it has been demonstrated to have bactericidal effects on Enterococcus faecalis which is the most resistant bacteria of the root canal system [26]. Though CHX is shown to act on the microscopic level, its clinical effect is not well demonstrated, according to Cochrane research database [27]. The clinical signs such as pain and swelling were not reduced with the use of CHX as an irrigant [26]. 2\% sodium hypochlorite is also an endodontist's preferred choice of root canal irrigant as it dissolves inorganic matter compared to CHX [28]. This characteristic feature of an irrigant is a significant one as it prevents failure of treatment. However, full-mouth disinfection with $0.2 \%$ CHX is preferred in endo-perio lesions where root canal treatment is completed [15].

\section{Oral surgery}

Post-operative infection of the extracted tooth site is one of the common complications of tooth extraction. CHX is effective in preventing this due to its anti-bacterial nature. Warm saline rinse stays as the first choice in this view. However, CHX when used as a pre-procedural rinse, it prevents post-operative bacteremia. In addition, Cochrane database suggests that CHX is highly effective for third molar extractions. This reduces the risk of alveolar osteitis following third molar extractions [29].

Medication-related osteonecrosis of the jaw (MRONJ), is a defect in the healing process seen with various medications such as bisphosphonates, RANKL inhibitors, and anti-angiogenic agents. Guidelines from England suggest using 0.2\% CHX mouthwash a week prior and up to 2 months after the removal of the tooth [30]. American Association of Oral and Maxillofacial Surgeons suggests using CHX mouthwash for the management of MRONJ [31]. However, Cochrane review concluded that more research is warranted to know more about CHX in the management or prevention of MRONJ [32].

Pre-procedural mouth rinse

Commonly used instruments in a dental operating field include ultrasonic scaler, aerator, and 3-way syringe, all of which are tend to produce aerosol infections. A moderate level of evidence is found to reduce bacterial aerosols when antiseptic solutions are used prior to the procedure [33]. When agar plates were placed in close proximity to the dental operating area (within 6 feet distance) $0.2 \%$ CHX mouth rinse prior to ultrasonic scaling reduced aerosol contamination by $70 \%$ [34]. Various studies have shown the use of herbal mouthwashes and other antiseptic solutions and their effectiveness in the reduction of aerosol production [35]. Hence, CDC guidelines suggest the use of CHX, essential oils, or Povidone-Iodine solution as a pre-procedural mouth rinse to reduce microbes in aerosol particles [35].

However, evidence is available only for bacterial aerosol contamination reduction. CHX is also known for its anti-viral properties, especially against enveloped viruses than non-enveloped ones [36]. Due to the high amount of availability of AngiotensinConverting Enzyme-2 receptor (ACE2) in the oral environment, the merging virus of COVID-19 pandemic Severe Acute Respiratory Syndrome Coronavirus-2 (SARS-CoV-2) resides in the oral cavity [37]. Though SARS-CoV-2 is an enveloped virus, the ability of CHX against viral loads in the oral cavity is still yet to be answered by research methods [38]. Hydrogen peroxide (1\%) mouth rinse is highly effective against viruses [39]. Hence, it should be consid- 
ered using during this pandemic period. Use of other mouthwashes such as Listerine, povidone-iodine, and herbal mouthwashes may also be effective against viral load in the oral cavity. Hence, more research is warranted in this direction.

\section{Other uses}

CHX has been effective against plaque formation and white spot lesions in fixed oral appliances such as orthodontic appliances, intermaxillary fixation devices [40]. Systematic review has concluded that CHX is weak in reducing dental caries in these patients, though streptococcus mutant level is reduced significantly [41]. The orthodontic society recommends using alcohol-free fluoride mouth wash against dental caries in fixed orthodontic patients [42]. CHX is not preferred by them as it could produce staining of teeth with long-term use. Hence, CHX is not indicated in such cases.

Maintenance of oral hygiene in head and neck cancer patients is at risk. Hence Royal College of Surgeons of England (RCS, Eng.) suggests using $\mathrm{CHX}$ as a plaque control agent in such cases [43].

Patients with special needs such as Down's syndrome, autism requires special attention in maintaining oral hygiene. The British Society of Periodontology (BSP) also advocates that the use of 'antiplaque agents like CHX are useful for managing acute periods when cleaning is difficult but not needed as a routine [44]. It should be kept in mind that CHX use is licensed for 30 days. 1\% CHX gel application reduces the potential development of periodontal diseases in Down's syndrome patients [45]. However, systematic review could not find any supporting evidence to state that the use of CHX reduces gingival inflammation in patients with special care needs [46]. Hence, the use of fluoride mouth rinse along with improvement in oral hygiene measures remains the first line of approach in maintenance of oral hygiene in such patients [46].

\section{Adverse effects and contraindications}

Despite the fact that CHX has a wide variety of uses in the dental field, it is not without adverse effects. Common adverse effects include teeth staining, xerostomia, and altered taste sensation for salt and bitter taste, increased calculus formation, and discolored tongue [47]. Other less common effects include swelling of the parotid gland, desquamation of oral mucosa, and burning sensation. Long-term use of CHX leads to teeth staining due to non-enzymatic browning (Maillard reaction) [48]. To prevent this reaction, the
CHX anti-discoloration system (CHX-ADS) is available now. The systematic review suggests that this newly developed product does not affect the effects of CHX in plaque control [49]. Type I and IV hypersensitivity reactions are the other common side effects seen with CHX use. Reports are available to show that CHX can lead to respiratory arrest and death due to anaphylactic reactions [50]. This is not related to any of the components of CHX mouthwash. In addition, CHX mouthwash may also be used in pregnancy. However, according to FDA (Food and Drug Agency), the use of Periochip is not advised in pregnancy and breastfeeding mothers as it does not follow safety limits in such patients [50]. Antimicrobial resistance is an emerging finding with CHX use [51]. This reduces the effectiveness of $\mathrm{CHX}$.

\section{Conclusion, Current Evidence and Future Directions}

To summarize, current evidence supports the fact that CHX may be effective against plaque formation, gingivitis, alveolar osteitis, bacterial aerosols, and symptomatic relief from viral-induced oral infections. Evidence is weak for the use of CHX against periodontitis, dental caries, ANUG, peri-implantitis, infections associated with tooth extraction, and virus aerosols. However, the use of CHX should always be considered with its adverse effects. More studies are recommended to further improve the level of evidence of CHX in the dental field.

\section{Bibliography}

1. Sajjan P., et al. "Chlorhexidine as an antimicrobial agent in dentistry-a review”. Oral Health and Dental Management (2016): 93-100.

2. Balagopal S and Radhika A. "Chlorhexidine: the gold standard antiplaque agent". Journal of Pharmaceutical Science and Research (2013): 270-274.

3. Poonam S., et al. "Chlorhexidine-an antiseptic in periodontics". Journal of Dental and Medical Sciences 13.9(2014): 85-88.

4. Jones CG. “Chlorhexidine: is it still a gold standard?". Periodontology 2000 (1997): 55-62.

5. Lang NP and Brecx MC. "Chlorhexidine digluconate-an agent for chemical plaque control and prevention of gingival inflammation". Journal of Periodontal Research (1986): 74-89. 
6. Emisilon C. "Susceptibility of various microorganisms to chlorhexidine". Scandinavian Journal of Dental Research (1977): 255-265.

7. P Gilbert and L Moore. "Cationic antiseptics: diversity of action under a common epithet". Journal of Applied Microbiology 99.4 (2005): 703-715.

8. F Cieplik., et al. "Resistance Toward Chlorhexidine in Oral Bacteria - Is There Cause for Concern?". Frontiers in Microbiology (2019): 587.

9. Wood D Payne. "The action of three antiseptics/disinfectants against enveloped and non-enveloped viruses". Journal of Hospital Infections (1998): 283-295.

10. Dehghani Nazhvani., et al. "Antifungal Effects of Common Mouthwashes on Candida Strains Colonized in the Oral Cavities of Liver Transplant Recipients in South Iran in 2014". Hepatitis Monthly (2016): e31245.

11. N P Lang., et al. "Plaque formation and gingivitis after supervised mouth rinsing with $0.2 \%$ delmopinol hydrochloride, $0.2 \%$ chlorhexidine digluconate and placebo for 6 months". Oral Diseases (1998): 105-113.

12. T Walsh., et al. "Chlorhexidine treatment for the prevention of dental caries in children and adolescents". Cochrane Database Systematic Review (2015): CD008457.

13. Y Wang., et al. "Effect of non-fluoride agents on the prevention of dental caries in primary dentition: A systematic review". PLoS One (2017): e0182221.

14. Scottish Dental Clinical Effectiveness Programme (SDCEP). "Drug Prescribing for- Dentistry". Evidence Based Dentistry (2016): 62.

15. Scottish Dental Clinical Effectiveness Programme (SDCEP). "Prevention and Treatment of Periodontal Diseases in Primary Care Dental Clinical Guidance". Evidence Based Dentistry (2014): 68-69.

16. M Sanz., et al. "Treatment of Stage I-III Periodontitis The EFP S3 Level Clinical Practice Guideline". Journal of Clinical Periodontology 22 (2020): 4-60.
17. Y Zhang., et al. "Chlorhexidine exposure of clinical Klebsiella pneumoniae strains leads to acquired resistance to this disinfectant and to colistin". International Journal of Antimicrobial Agents 53.6 (2019): 864-867.

18. J Kolahi and A Soolari. "Rinsing with chlorhexidine gluconate solution after brushing and flossing teeth: a systematic review of effectiveness". Quintessence International (2006): 605-612.

19. D Herrera., et al. "Adjunctive effect of locally delivered antimicrobials in periodontitis therapy. A systematic review and meta-analysis". Journal of Clinical Periodontology (2020): 239256.

20. P James., et al. "Chlorhexidine mouth rinse as an adjunctive treatment for gingival health". Cochrane Database Systematic Review (2017): CD008676.

21. J Eberhard., et al. "Fullmouth disinfection for the treatment of adult chronic periodontitis". Cochrane Database Systematic Review (2008): CD00462.

22. CV do Amori., et al. "Susceptibility of some oral microorganisms to chlorhexidine and paramonochlorophenol". Brazilian Oral Research (2004): 242-246.

23. G Bryce., et al. "Pre- and post-operative management of dental implant placement. Part 2: management of early-presenting complications". British Dental Journal 217.4 (2014): 171-176.

24. F Schwarz., et al. "Efficacy of alternative or adjunctive measures to conventional treatment of peri-implant mucositis and periimplantitis: a systematic review and meta-analysis". International Journal of Implant Dentistry 1.1 (2015): 22.

25. YC de Waal., et al. "Implant decontamination with 2\% chlorhexidine during surgical peri-implantitis treatment: a randomized, double-blind, controlled trial". Clinical Oral Implants Research 26.9 (2015): 1015-1023.

26. L Mallya., et al. "Evaluation of the antimicrobial efficacy of $20 \%$ Punica granatum, $0.2 \%$ chlorhexidine gluconate, and $2.5 \%$ sodium hypochlorite used alone or in combinations against Enterococcus faecalis: An invitro study". Journal of Conservative Dentistry 22.4 (2019): 367-370. 
27. LS Gonçalves., et al. "The Effect of Sodium Hypochlorite and Chlorhexidine as Irrigant Solutions for Root Canal Disinfection: A Systematic Review of Clinical Trials". Journal of Endodontics 42.4 (2016): 527-532.

28. M Haapasalo., et al. "Irrigation in endodontics". British Dental Journal 54.2 (2014): 299-303.

29. Daly., et al. "Local interventions for the management of alveolar osteitis (dry socket)". Cochrane Database Systematic Review 12 (2012): CD006968.

30. NHS England. "Dental Management of Patients Prescribed Bisphosphonates - Clinical Guidance”. (2015).

31. SL Ruggiero., et al. "American Association of Oral and Maxillofacial Surgeons position paper on medication-related osteonecrosis of the jaw-2014 update". Journal of Oral Maxillofacial Surgery 72.10 (2014): 1938-1956.

32. V Rollason., et al. "Interventions for treating bisphosphonaterelated osteonecrosis of the jaw (BRONJ)". Cochrane Database Systematic Review 2.2 (2016): CD008455.

33. VC Marui., et al. "Efficacy of preprocedural mouthrinses in the reduction of microorganisms in aerosol: A systematic review". The Journal of the American Dental Association 150.12 (2019): 1015-1026.e1.

34. G Gupta., et al. "Efficacy of preprocedural mouth rinsing in reducing aerosol contamination produced by ultrasonic scaler: a pilot study". Journal of Periodontology 85.4 (2014): 562-568.

35. WG Kohn., et al. "Guidelines for Infection Control in Dental Health-Care Settings". Morbidity and Mortality Weekly Report (2003): 521-561.

36. Farzan P Firoozi. "Common Mouthwashes for Pre-procedural Rinsing in Dental Practice: Which One is Appropriate for Eliminating Coronaviruses? A Mini Literature Review". Journal of Regeneration, Reconstruction and Restoration (2020): e2.

37. H Xu., et al. "High expression of ACE2 receptor of 2019-nCoV on the epithelial cells of oral mucosa". International Journal of Oral Science (2020): 8.
38. JG Yoon., et al. "Clinical Significance of a High SARS-CoV-2 Viral Load in the Saliva". Journal of Korean Medical Science 35.20 (2020): e195.

39. X Peng., et al. "Transmission routes of 2019-nCoV and controls in dental practice". International Journal of Oral Science (2020): 9.

40. Gehlen., et al. "The influence of a $0.2 \%$ chlorhexidine mouthrinse on plaque regrowth in orthodontic patients. A randomized prospective study. Part II: Bacteriological parameters". Journal of Orofacial Orthopedics 61.2 (2000): 138-148.

41. X Tang., et al. "The antimicrobial effect of chlorhexidine varnish on mutans streptococci in patients with fixed orthodontic appliances: a systematic review of clinical efficacy". International Journal of Dental Hygiene 14.1 (2016): 53-61.

42. British Orthodontic Society Patient Leaflet. "Fixed Appliances" (2020).

43. N Kumar., et al. "The oral management of oncology patients requiring radiotherapy, chemotherapy and/or bone marrow transplantation". Faculty Dental Journal 4.4 (2013): 200-203.

44. British Periodontal Society. "The Good Practitioner's Guide to Periodontology". $3^{\text {rd }}$ Ed. (2016).

45. The Royal College of Surgeons of England. "Clinical Guidelines and Integrated Care Pathways for the Oral Health Care of People with Learning Disabilities" (2012).

46. N Zhou., et al. "Efficacy of caries and gingivitis prevention strategies among children and adolescents with intellectual disabilities: a systematic review and meta-analysis". Journal of Intellectual Disability Research 63.6 (2019): 507-518.

47. FB Zanatta., et al. "Staining and calculus formation after $0.12 \%$ chlorhexidine rinses in plaque-free and plaque covered surfaces: A randomized trial". Journal of Applied Oral Sciences 18.5 (2010): 515-521.

48. GM Tartaglia., et al. "Adverse events associated with home use of mouthrinses: a systematic review". Therapeutic Advances in Drug Safety 10 (2019): 2042098619854881. 
49. BWM Van Swaaij., et al. "Does chlorhexidine mouthwash, with an anti-discoloration system, reduce tooth surface discoloration without losing its efficacy? A systematic review and metaanalysis". International Journal of Dental Hygiene 18.1 (2020): 27-43.

50. MN Pemberton and J Gibson. "Chlorhexidine and hypersensitivity reactions in dentistry". British Dental Journal 213.11 (2012): 547-550.

51. G Kampf. "Acquired resistance to chlorhexidine - is it time to establish an' antiseptic stewardship' initiative?". Journal of Hospital Infections 94.3 (2016): 213-227.

\section{Volume 5 Issue 11 November 2021}

(C) All rights are reserved by Valliammai Rajendran., et al. 